\title{
Fractional Feature Extraction and Pattern Recognition of Transformer Partial Discharge Sources
}

\author{
WANG Guo-qiang, ZHU Yong-li, LIU Li-peng \\ Department of Electrical Engineering \\ North China Electric Power University \\ Baoding, China \\ 834819987@qq.com
}

\begin{abstract}
The recognition of transformer partial discharge patterns is helpful for evaluating insulation condition of transformers.Six typical kinds of partial discharge patterns are tested in the study.Based on the characteristics of the data,the partial discharge patterns were divided into several classes. Thus the pattern recognition can be translated into a two category process,in which the recognition can be realized gradually.By analyzing the data,the characteristic parameters are found for recognizing the classes.Then use the confidence interval method to realize the recognition process, and the accuracy of this method was verified.The results show that the method is reliable for the partial discharge pattern recognition.
\end{abstract}

Keywords-transformer; partial discharge; feature extraction; recognition

\section{INTRODUCTION}

The good insulation condition is the necessary conditions of the power transformer safe operation, and partial discharge can cause transformer insulation degradation. Partial discharge pattern recognition is helpful to judge the severity of insulation degradation and the position of partial discharge . Therefore, it is very meaningful to find a method which can realize partial discharge pattern recognition simply and effectively.

Because of the difference of each partial discharge modes in structure and material, the various statistical spectrum charts ${ }^{[1]}$ have the characteristics respectively. Some statistical operators $^{[2-3]}$ were found to reflect the characteristics of PD models, and the pattern recognition can be realized by the differences of the statistical operators. Through analyzing these statistical operators, only some of them have clearly distinctions in numerical values between two certain PD models, and others'taking value intervals are overlap which is adverse for recognition of PD models. The existing recognition methods often ignore these.They always compare all the characteristics at one time. So not only the accuracy of the results are reduced,but the calculation quantity is increased.

Therefore the paper select the operators whose dipartite degree is apparent according to the partial discharge types .It make PD patterns simple and effective to distinguish from each other by summarizing the numerical differences of the operators .

\section{THE SYSTEM OF THE TEST AND THE MEASUREMENT}

\section{A. The Measurement System}

The test uses the standard IEC60270-2000 $0^{[4]}$. The circuit is the parallel test circuit based on pulse current method.The model of the PD integrated analyzer is TWPD-2F, the simpling frequency is $5 \mathrm{MHz}$, the bandwidth is $40 \mathrm{k} 300 \mathrm{kHz}$ 。 The test platform model is TWI513310/100am,the transformer model used is YD0-10100。

Tune test voltage up until the PD pulses is stable and pulses amplitude is obviously. Test data in a power frequency was recorded as a group.Each of $\operatorname{Hqn}(\varphi), \operatorname{Hn}(\varphi)$ and $\operatorname{Hqmax}(\varphi)$ was obtained by counting 50 groups of data. The pulse count distribution $\mathrm{Hn}(\mathrm{p})$, which represents the number of observed discharges in each phase window as a function of the phase angle. The mean pulse height distribution $\operatorname{Hqn}(\mathrm{p})$, which represents the average amplitude in each phase window as a function of the phase angle.The max pulse height distribution $\operatorname{Hqmax}(\varphi)$, which represents the max amplitude in each phase window as a function of the phase angle.

\section{B. The Structure of the Samples}

There are internal discharge models,suspended discharge models,needle-plate discharge models and surface discharge models in the test.The structure of the models were shown as paper [5-6].

The internal discharge models has two types. One kind of them is the void enclosed in paperboard;in other type,the void is located in the interface between the paperboard and the electrode. There are two surface discharge models in the test, one in air and the other in oil. 


\section{FEATURE EXTRACTION}

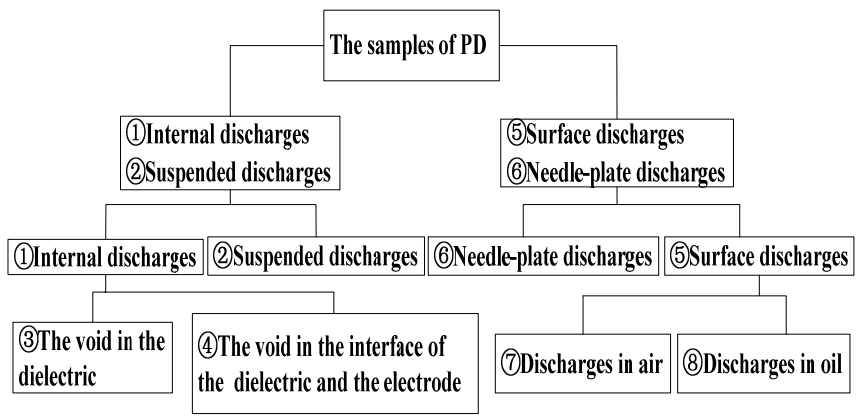

Figure 1. Recognition process of discharge patterns.

According to the feature of the types of PD, the paper design a recognition process shown as Figure.1.Each group of characteristic parameters are extracted aiming at each two types of models and the characteristics should have a good dipartite degree.If every two classes can be distinguished exactly, the recognition of PD models can be realized.

\section{A. Classification Based on the Phase Distribution}

In the first step, make the internal discharge and suspended discharge as a group and needle-plate discharge and surface discharge as a group.In internal discharge and suspended discharge,the speed of charge lapse is very low,because of the conductor suspending or the insulation around the void.So it is easy for charge to accumulate in the defect.Thus the charge would produce a electric field whose direction is opposite to the test applied voltage.It would reduce the total field strength. Therefore the PD pulses appear when the applied voltage rise, and there are no pulses appearing in the phase after the peak of voltage.However ,in other two types of discharge, the pulses distribute two side of the voltage peak symmetrically, because the charge produced by discharge disappear more quickly in these models.

In the paper, choice the phase median to reflect the characteristic mentioned above.The phase median $\mathrm{Mv}$ is obtained as follow.

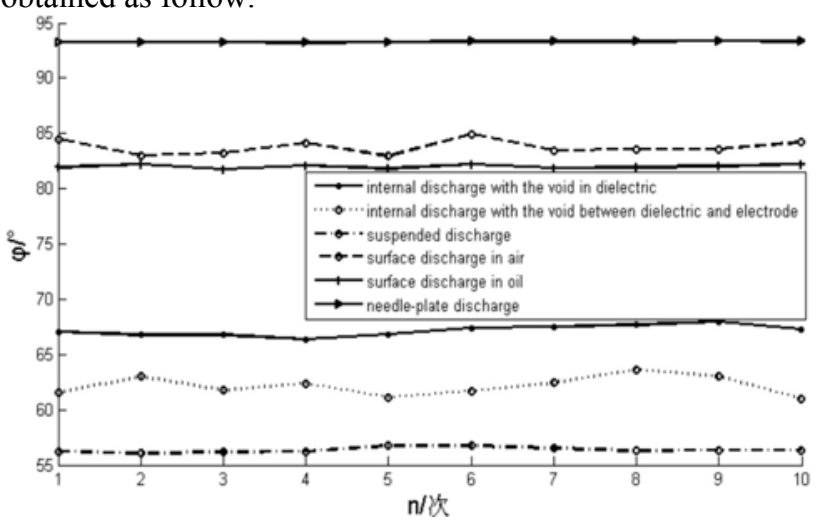

Figure 2. Phase median of the discharge models.

It should be took into consideration that discharge can happen around the zero point of applied voltage ${ }^{[4]}$. So set [- $\left.15^{\circ}, 165^{\circ}\right)$ as the positive half of the voltage cycle and $\left(165^{\circ}, 345^{\circ}\right]$ as the negative half of the voltage cycle.

$$
M v=\frac{M v^{+}+\left(M v^{-}-180\right)}{2}
$$

The superscripts of $\mathrm{Mv}^{+}$and $\mathrm{Mv}^{-}$indicate respectively the positive and the negative half of the voltage cycle.The superscripts in other places also have the same meaning.

Figure. 2 shows the phase medians are stable during the test.It is suitable as the characteristic to complete classing of the first step.

\section{B. The Difference of Internal Discharge and Suspended Discharge}

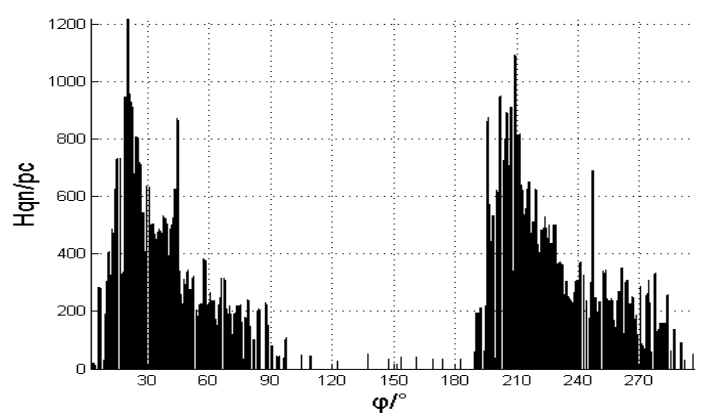

Figure 3. Hqn $(\varphi)$ patterns of internal discharges.

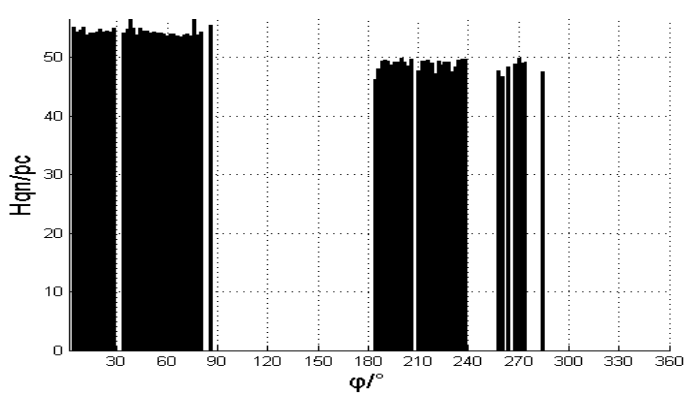

Figure 4. Hqn $(\varphi)$ patterns of suspended discharges.

In the test,the amplitude fluctuation of suspended discharge is not obvious.But internal discharge is not same as suspended discharge.This characteristic can be observed from figure 3 and figure 4.

The statistical data shows the value range of the two types of discharge is different from each other. And the difference is more appea in the positive half of the voltage cycle.In the test,the skewness of suspended discharge's $\mathrm{H}_{\mathrm{qn}}(\varphi)^{+}$is in the value interval $(-0.33,0.22)$; the kurtosis is in the value interval $(-1.7,-1.1)$, while the skewness and kurtosis of internal charge is greater than that of suspended discharge apparently. 
C. The Difference of Needle-Plate Discharge and Surface Discharge

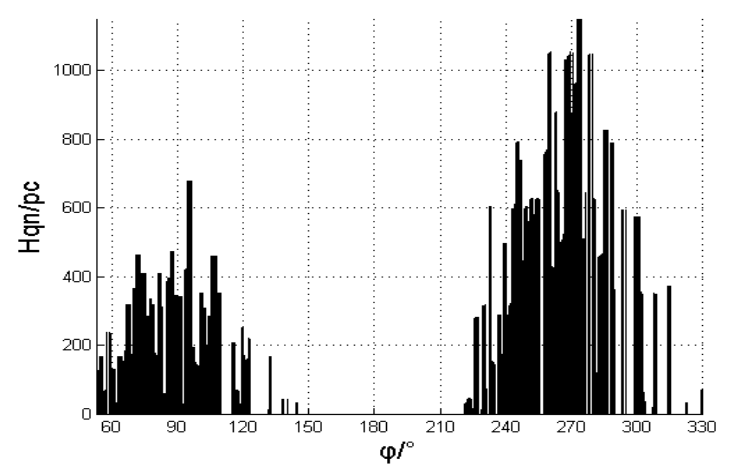

Figure 5. $\operatorname{Hqn}(\varphi)$ patterns of needle plate discharges.

Needle-plate discharge is belong to a asymmetric electrode system.Its discharge occurred around the electrode. The polarity effect is very obvious and it is clearly there is asymmetry between the positive and the negative half of the voltage cycle.As shown in figure 5 to figure 7,needle-plate discharge is more apparent than surface discharge in the asymmetry between the positive and negative discharge.

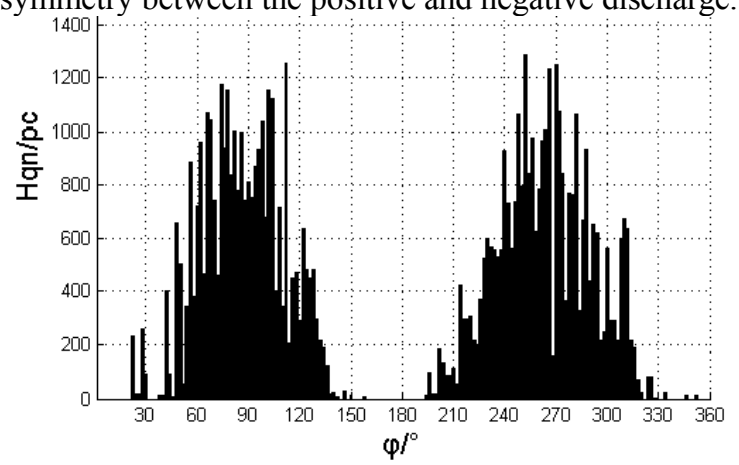

Figure 6. Hqn $(\varphi)$ patterns of surface discharges in air.

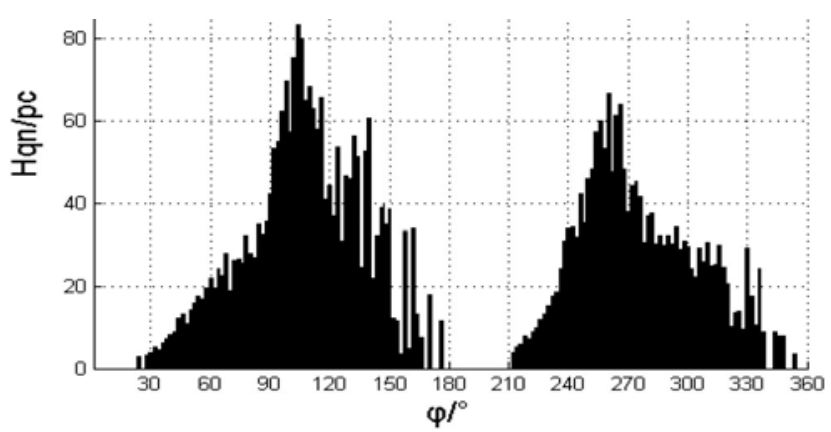

Figure 7. Hqn $(\varphi)$ patterns of surface discharges in oil.

For reflecting the difference between the two types of partial discharge, this paper introduces a modified correlation coefficient mcc,defined as follows:

$$
Q=\frac{\min \left(Q_{\max }^{+}, Q_{\max }^{-}\right)}{\max \left(Q_{\max }^{+}, Q_{\max }^{-}\right)}
$$

$$
\begin{gathered}
\Phi=\frac{\min \left(\varphi_{i n}^{+}, \varphi_{i n}^{-}\right)}{\max \left(\varphi_{i n}^{+}, \varphi_{i n}^{-}\right)} \\
m c c=Q \cdot \Phi \cdot c c
\end{gathered}
$$

The $\mathrm{Q}_{\max }$ is the maximum discharge magnitude ; the $\mathrm{Q}_{\min }$ is the minimal discharge magnitude;cc is cross-correlation coefficient of the positive and the negative half of the voltage cycle.They can be retained as the definition of the paper[5].Higher the mcc is,more asymmetric the discharge of the positive and the negative half of the voltage is.The mcc $=1$ means a complete symmetric and a value of 0 indicates total asymmetric.Though analyzing the date recorded in the test,it is less than 0.45 that the mcc of $\mathrm{Hn}(\varphi)$ from needle-plate discharge, but it is above 0.60 in surface discharge.So mcc is suitable for the distinguishing of this two types of partial discharge models.

D. The Difference of the Void in the Dielectric and the Void in the Interface Between the Dielectric and Electrode

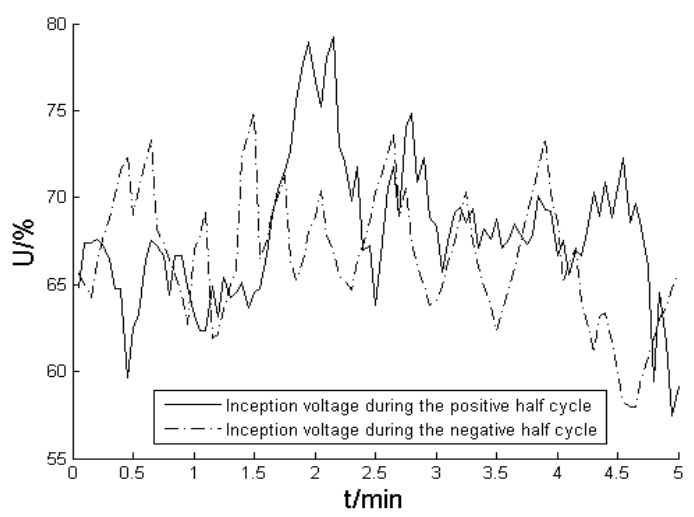

a. Air gap in dielectric.

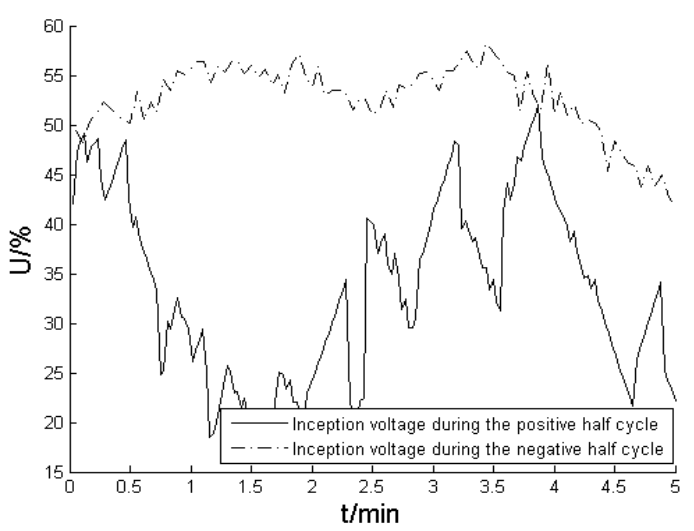

b. Air gap between electrode and dielectric

Figure 8. Inception voltage of internal discharges.

The internal discharge models can be divided into two types (3)and (4)according to the position of the voids.If the electrode contacting the void is ground potential,the effective initiation electrons have to be freed from the electrode or natural background radiation during the positive half 
cycle.However,in the negative half cycle ,there are larges of initiation electrons supplying because of the remnant charge at the dielectric surface.So the inception voltage is very stable in the negative half cycle,while a fluctuant inception voltage is in positive half cycle,shown as in figure 8.The variance can reflect the difference well.

Calculate the variance $\mathrm{S}^{+}$of the positive half cycle and the variance $\mathrm{S}^{-}$of the positive half cycle respectively. Then calculate the ratio as follow.

$$
C=\frac{\max \left(S^{+}, S^{-}\right)}{\min \left(S^{+}, S^{-}\right)}
$$

The $\mathrm{C}$ of the model (3) is less than 2,and the $\mathrm{C}$ of the model (4) is above 5 . The value of $\mathrm{C}$ can be used to classify the models (3) and (4).

\section{E. The Difference of Surface Discharge in Oil and in Air}

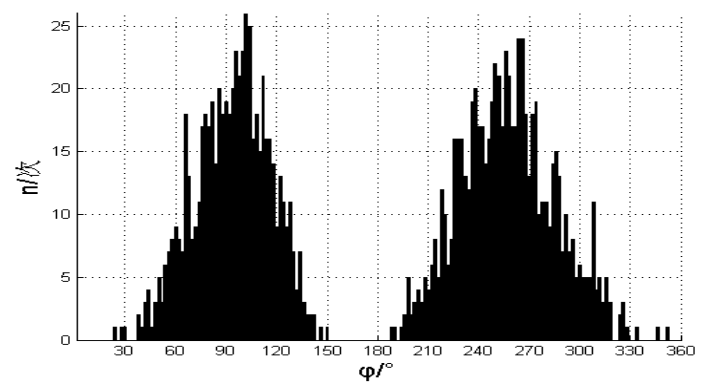

Figure 9. $\operatorname{Hn}(\varphi)$ patterns of surface discharges in air.

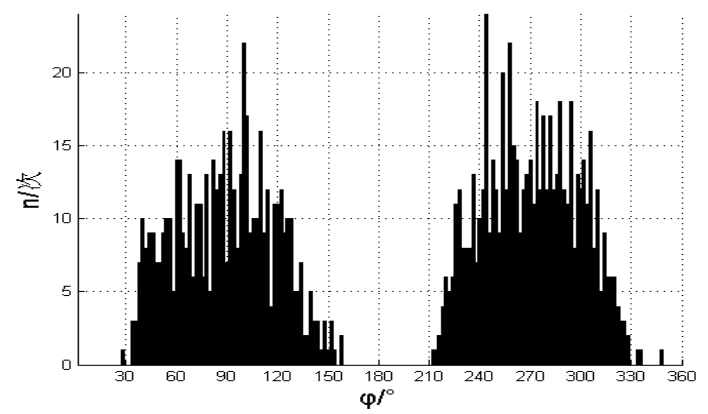

Figure 10. $\operatorname{Hn}(\varphi)$ patterns of surface discharges in oil.

Oil and air have different effect on the emergence and develop of discharge $\mathrm{e}^{[7-8]}$ and it is very obvious in the atlas of $\operatorname{Hn}(\varphi)$.The skewness can reflect this characteristic.Specifically, the sk is all above 0.15 in the air,and the sk value of surface discharge in oil is near the zero, less than 0.10 .

\section{THE RECOGNITION OF PARTIAL DISCHARGE MODELS}

Based on the conclusion of the last chapter,the recognition process shown in figure 11 is adopted.The test shows that every characteristic used in each step has a very good distinguish degree.So this paper uses the confidence interval method ${ }^{[6]}$ to achieve the model recognition.Calculate the corresponding characteristic value and the corresponding probability,set the one whose probability is higher as the
result.If there are two characteristics in a step, it is compared that the sum of the two probabilities,setting the higher one as the result.

The mean and standard deviation of every confidence interval are drawn from 60 samples, choosing the confidence level of $99 \%$.Considering the time getting the $\mathrm{C}$ is long and the $\mathrm{C}$ of (3) and (4) is very different, a boundary value is setted ; greater than the value is belong to (4) and it is the type of (3) if less than the value.Then choose other 30 samples in every type of model to test the recognition process, only one sample of needle-plate charge is classified in a error type and other result are all correct.

In order to getting further verification, the paper tested the data from discharge in air gap between two wire with insulation and the corona discharge, and the results of recognition are internal charge whose void is in the dielectric and needle-plate discharge.It presented the method is not only simple and intuitive, but have high identification accuracy.

\section{CONCLUSION}

a) The partial discharge pulse distribution can reflect the loss rate of charge in defects at some extent.The condition around the defects can be generally reflected from the pulse distribution.The atlases of the positive and the negative half cycle is different obviously in the asymmetric electrode system.This characteristic is very useful in distinguishing the asymmetric electrode system from the asymmetric one.

b) The recognition of partial discharge can be realized by the process of gradual classifying.It is convenient for the extraction of the characteristics based on the content of every step. In this paper,the method is proved to be helpful in simplifying the identification and improving the accuracy of classifying.

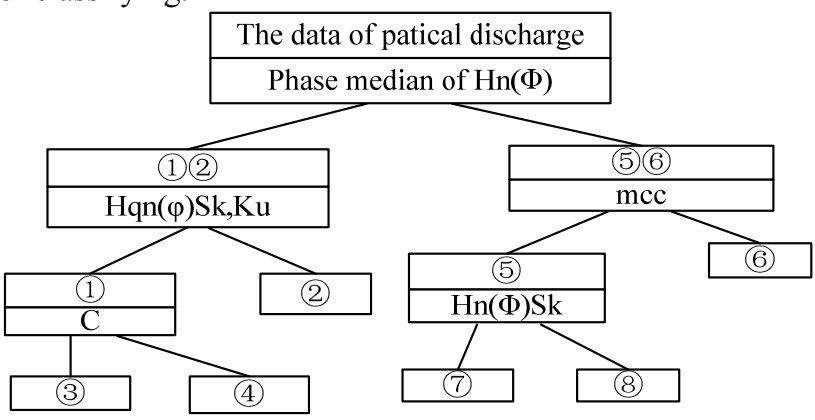

Figure 11. The classification process of the discharge modes.

\section{REFENCE}

[1] GUO Jun ,WU Guang-ning,ZHANG Xue-qin,et al. The actuality and perspective of partial discharge detection techniques[J].Transactions of China Electrotechnical Society,2005,20(2):29-35.

[2] Gulski E,Kreuger F H. Computer-aided recognition of discharge sources[J]. IEEE Trans. on Electrical Insulation, 1992, 27(1) : 82-92.

[3] ZHU De-heng,YAN Zhang,TAN Ke-xiong,et al.Condition Monitoring and Failure Diagnose Technology of Electrical Equipment[M].Beijing:China Electric Power Press,2009.112-114. 
[4] International Electrotechnical Commission.IEC60270-2000 Partial discharge measurements[S].Geneva:International Electrotechnical Commission, 2000.

[5] WU Peng,CHEN Zhi-yong,Li Zhi-yong,et al.Experimental research on typical PD models for power Transformers.High Voltage Apparatus,2004,40(3):161-163.

[6] Li Yan-qing,CHEN Zhi-ye,LV Fang-cheng,et al. Pattern recognition of transformer partial discharge based on acoustic method.Proceedings of the CSEE,2003,23(2):108-111.

[7] WANG Guo-li,YUAN Peng,SHAN Ping,et al.Automatic identification system of transformer partial discharge using ultra high frequency $[\mathrm{J}]$. Advanced Technology of Electrical Engineering and Energy,2003,22(1): 9-13.

[8] ATTEN P,SAKER A.Streamer propagation over a liquid/solid interface[J].IEEE Trans on Electrical Insulation, 1993,28(2):230-242. 